\title{
Cannabinoid exposure during zebra finch sensorimotor vocal learning persistently alters expression of endocannabinoid signaling elements and acute agonist responsiveness
}

\author{
Ken Soderstrom ${ }^{1 *}$, Justin L Poklis², Aron H Lichtman²
}

\begin{abstract}
Background: Previously we have found that cannabinoid treatment of zebra finches during sensorimotor stages of vocal development alters song patterns produced in adulthood. Such persistently altered behavior must be attributable to changes in physiological substrates responsible for song. We are currently working to identify the nature of such physiological changes, and to understand how they contribute to altered vocal learning. One possibility is that developmental agonist exposure results in altered expression of elements of endocannabinoid signaling systems. To test this hypothesis we have studied effects of the potent cannabinoid receptor agonist WIN55212-2 (WIN) on endocannabinoid levels and densities of $\mathrm{CB}_{1}$ immunostaining in zebra finch brain.

Results: We found that late postnatal WIN treatment caused a long-term global disregulation of both levels of the endocannabinoid, 2-arachidonyl glycerol (2-AG) and densities of $\mathrm{CB}_{1}$ immunostaining across brain regions, while repeated cannabinoid treatment in adults produced few long-term changes in the endogenous cannabinoid system.

Conclusions: Our findings indicate that the zebra finch endocannabinoid system is particularly sensitive to exogenous agonist exposure during the critical period of song learning and provide insight into susceptible brain areas.
\end{abstract}

\section{Background}

Zebra finches learn a song during distinct periods of vocal development [1]. Exposure to cannabinoid agonists during these periods alters vocal development by reducing both song stereotypy and the number of notes incorporated into mature song produced in adulthood $[2,3]$. This, combined with evidence for distinct developmental regulation of $\mathrm{CB}_{1}$ cannabinoid receptor expression during periods of song learning [4], suggests a role for endogenous cannabinoid signaling in normal vocal developmental processes. Vocal learning and production in zebra finches is associated with marked physiological changes within distinct regions of telencephalon (e.g. IMAN, Area X, auditory Field L2, RA) and thalamus

\footnotetext{
* Correspondence: soderstromk@ecu.edu

'Department of Pharmacology and Toxicology, Brody School of Medicine,

East Carolina University, Greenville, NC 27834, USA

Full list of author information is available at the end of the article
}

(DLM, ovoidalis) known to be critical for song perception, production and learning. Each of these regions distinctly and densely expresses $\mathrm{CB}_{1}$ receptors [3]. Normal development in song regions is associated with gross anatomical changes in region volume, neuron number and density, both increases and decreases in axonal interconnections between song regions, and changes in synaptic densities. Cannabinoid-altered vocal development implies that exogenous agonist exposure must somehow alter some or all of these processes responsible for critical periods of song learning. We are currently working to identify which processes are modified by developmental cannabinoid exposure and the mechanism (s) responsible.

Distinct song region $\mathrm{CB}_{1}$ receptor expression implies a role for endocannabinoid signaling in vocal learning. The endocannabinoid system is a bona fide neurochemical signaling system comprising at least two 
G-protein-coupled receptors $\left(\mathrm{CB}_{1}\right.$ and $\mathrm{CB}_{2}$, with the former expressed at much higher density in CNS), and an array of fatty acid ligands, most notably anandamide and 2-arachidonyl glycerol (2-AG), that are capable of activating the receptors. Like most cell signaling systems, cannabinoid receptors and endocannabinoid ligands are subject to biochemical regulation of synthesis, expression and metabolic breakdown [5]. A particularly notable form of G-protein-coupled receptor regulation is agonistinduced internalization and metabolism, whereby the duration of agonist effects are limited by decreased receptor expression [6]. Thus, a potential mechanism for exogenous cannabinoid-induced alteration of normal development may involve endocannabinoid and receptor regulation. This hypothesis was tested through the experiments described below.

\section{Results \\ $\mathrm{CB}_{1}$ immunostaining}

Relative optical densities of $\mathrm{CB}_{1}$ immunostaining within selected brain regions are summarized by treatment groups in Figures 1 and 2. Persistent effects of chronic, 25-day WIN treatments on $\mathrm{CB}_{1}$ staining densities were assessed by comparing VEH-VEH and WIN-VEH groups. In the case of animals treated during sensorimotor development (Figure 1), repeated daily WIN treatments led to a significant decrease in staining density within all regions except DLM (differences from VEH$\mathrm{VEH}$ group indicated by an asterisk). This contrasts with chronic treatment effects produced in adult animals where chronic WIN treatments either produced no effect (IMAN, Area X, Ov, cerebellum) or increased staining densities (HVC, RA, DLM, see Figure 2).

Acute effects of WIN treatments (given 90 min prior to perfusion) on staining densities were assessed by comparing VEH-VEH and VEH-WIN groups. Following developmental vehicle injections and maturation to early adulthood, acute WIN treatments decreased $\mathrm{CB}_{1}$ immunostaining densities in all brain regions except DLM. A different pattern of acute responsiveness was observed in animals treated in adulthood: acute increases in staining densities were observed within HVC, RA, DLM and Ov. Acute responsiveness following repeated WIN administration was assessed by comparing WIN-VEH to WIN-WIN groups. In the case of animals treated with WIN during development, acute WIN exposure resulted in significantly increased $\mathrm{CB}_{1}$ immunostaining densities in all brain regions studied (Figure 1, differences indicated by a double-dagger). This effect of acute WIN to increase staining densities following chronic WIN differed with responses observed following chronic VEH in every brain region (Figure 1, compare VEH-WIN to WIN-WIN, differences indicated by a dagger). Following repeated WIN treatments of adults, no acute WIN effect was detected in any region except within the molecular layer of cerebellum where densities were increased following earlier chronic WIN exposure (Figure 2G, compare WIN-VEH to WIN-WIN, difference indicated by a double-dagger).

\section{Effects on brain 2-AG levels}

Effects of repeated WIN treatments on levels of the endocannabinoid 2-AG in various brain regions were assessed by LC-ESI-MS-MS. Results are summarized in Figure 3. WIN exposure during sensorimotor development significantly increased 2-AG levels in rostral telencephalon (containing the song regions IMAN and Area $\mathrm{X}$, Figure 3A). Levels were unchanged in caudal telencephalon (containing vocal motor regions, HVC and RA) and thalamus/midbrain (containing Ov and DLM). In contrast to effects produced in rostral telencephalon, developmental WIN exposure decreased 2-AG content within cerebellum. No effects of repeated WIN treatments on 2-AG levels in adult animals were observed in any of the brain regions studied (Figure 3B).

\section{Discussion}

Results reported herein demonstrate that developmental cannabinoid exposure persistently disregulates endocannabinoid signaling at both the receptor and endogenous agonist levels. This disregulation is significant because it's accompanied by altered vocal development that is produced by the same cannabinoid treatments [2,7]. These findings are among the first to associate cannabinoid-altered behavioral development with persistentlyaltered neurophysiology.

More work in evaluating persistent cannabinoid effects has employed cell and tissue culture systems. For example, in ex vivo cultures of rat hippocampi, periods of $24 \mathrm{hr}$ WIN exposure dose-dependently reduces $\mathrm{CB}_{1}$ immunostaining [8] which is consistent with persistent effects of chronic WIN that we find after developmental, but not adult exposure (compare VEH-VEH and WINVEH groups, Figures 1 and 2). It may be important that most ex vivo tissue culture systems employ tissue obtained from immature animals. For example, in the case cited above, hippocampi were isolated from rat pups at post-natal day two. Therefore, ex vivo cultures of CNS tissue may more closely model effects on the developing CNS than the mature, adult system. Interestingly, rat and zebra finch development occur over similar periods, with 'adolescence' reached between 35 - 45 days, and early adulthood by about 90 days $[7,9]$.

In cultures of cells heterologously expressing $\mathrm{CB}_{1}$ receptors, it has been appreciated for some time that agonist stimulation promotes a rapid internalization of receptor protein [10]. In our histological model, tissue is permeabilized, allowing antibody access to both plasma 


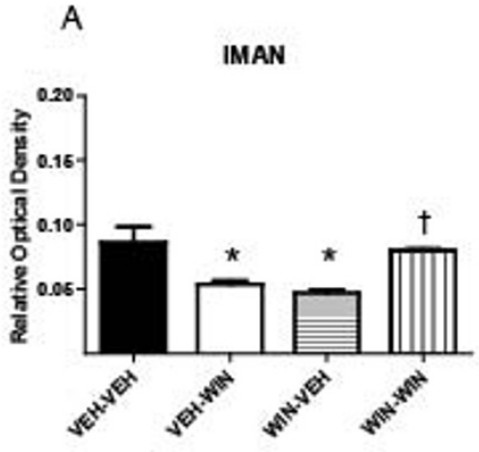

C

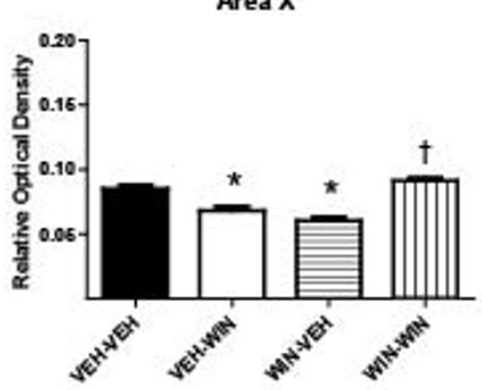

E

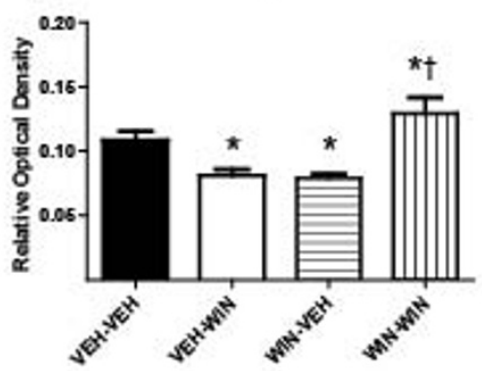

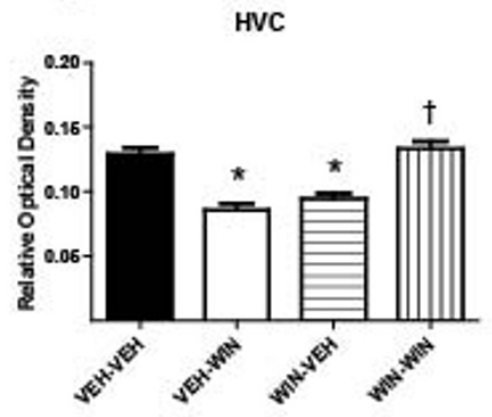

D

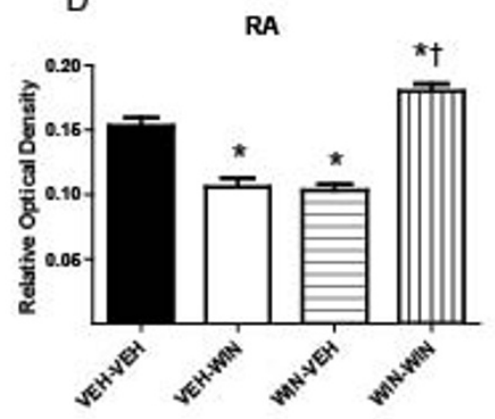

$\mathrm{F}$

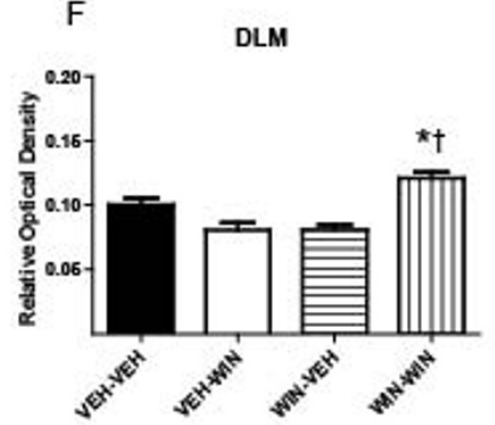

G Cerebellum

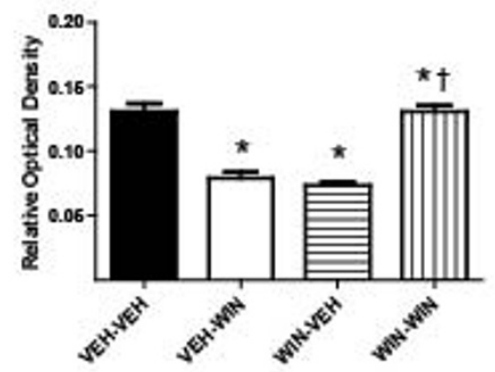

Figure 1 Developmental cannabinoid exposure alters $\mathrm{CB}_{1}$ immunostaining within various zebra finch brain regions. Initial daily treatments over 25 days are indicated by first designations (VEH-, WIN-). Later, single acute treatments in adulthood are indicated second (-VEH, -WIN, see Table 1). Treatments were delivered during sensorimotor song learning (from 50-75 days) and measured in adulthood (> 100 days). Basal levels of staining are decreased following repeated WIN exposure during development in all regions but DLM (compare VEH-VEH to WINVEH). Acute responsiveness is increased in all regions (compare VEH-WIN to WIN-WIN). Asterisks indicate differences from VEH-VEH treatment groups ( $p<0.05$, one-way ANOVA followed by SNK post-tests). Daggers indicate differences from VEH-WIN groups, double-daggers indicate differences from WIN-VEH. 


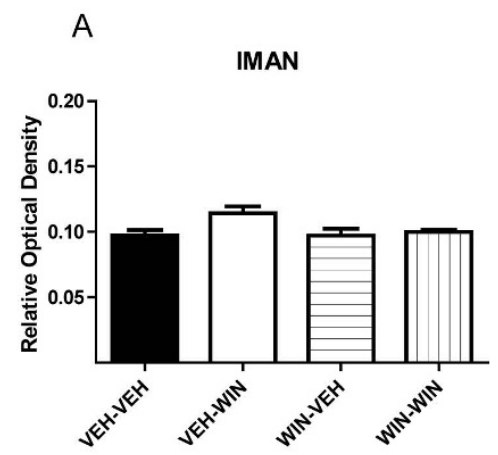

C

Area $\mathbf{X}$

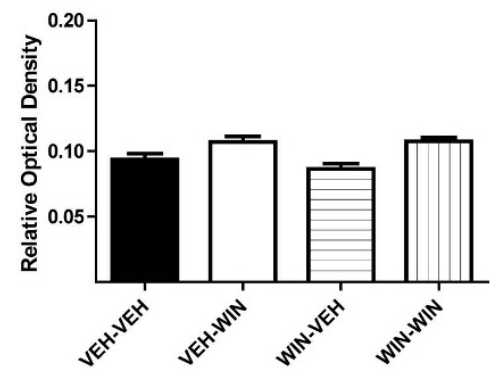

E

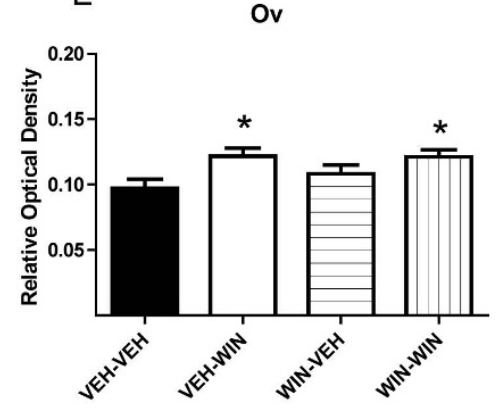

B

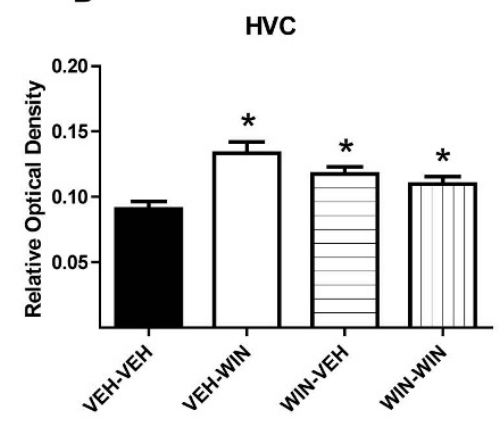

D

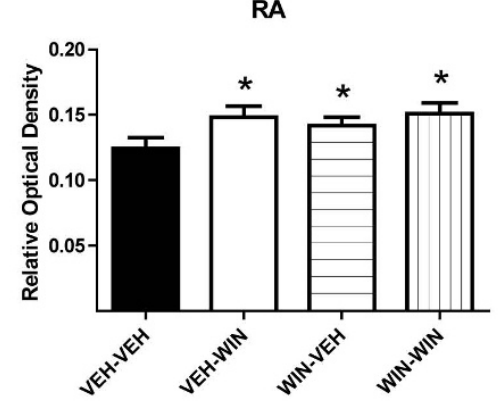

$\mathrm{F}$
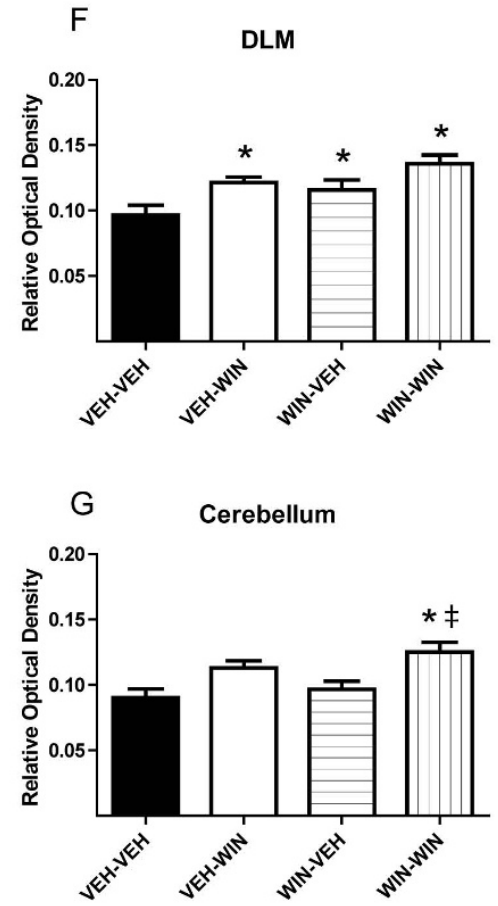

Figure 2 Repeated cannabinoid treatment during adulthood alters $\mathrm{CB}_{1}$ immunostaining primarily within vocal-motor-related regions of zebra finch brain (HVC, RA, and DLM). Initial daily treatments over 25 days are indicated by first designations (VEH-, WIN-). Later, single acute treatments are indicated second (-VEH, -WIN, see Table 1). Basal staining levels are increased following repeated WIN exposure in adulthood in (B) HVC, (D) RA, and (F) DLM (compare VEH-VEH to WIN-VEH in these panels). Acute responsiveness is not modified following repeated treatments (compare VEH-WIN and WIN-WIN). Chronic treatment did increase responsiveness within the molecular layer of the cerebellum (double-dagger, panel G) Asterisks indicate differences from VEH-VEH treatment groups $(p<0.05$, one-way ANOVA followed by SNK post-tests). 


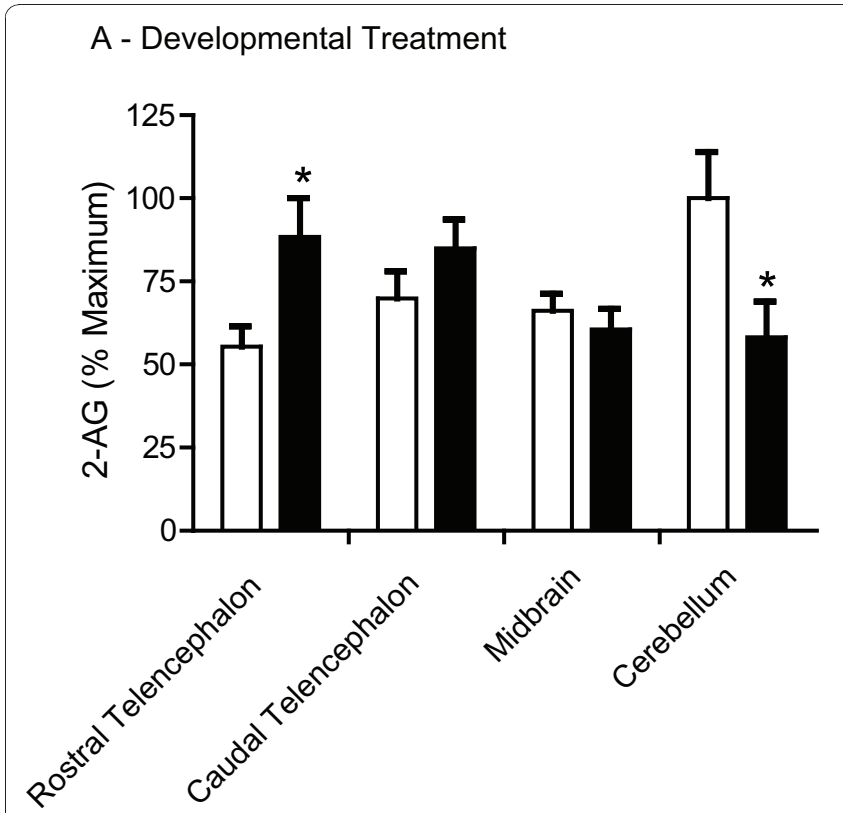

\section{B - Adult Treatment}

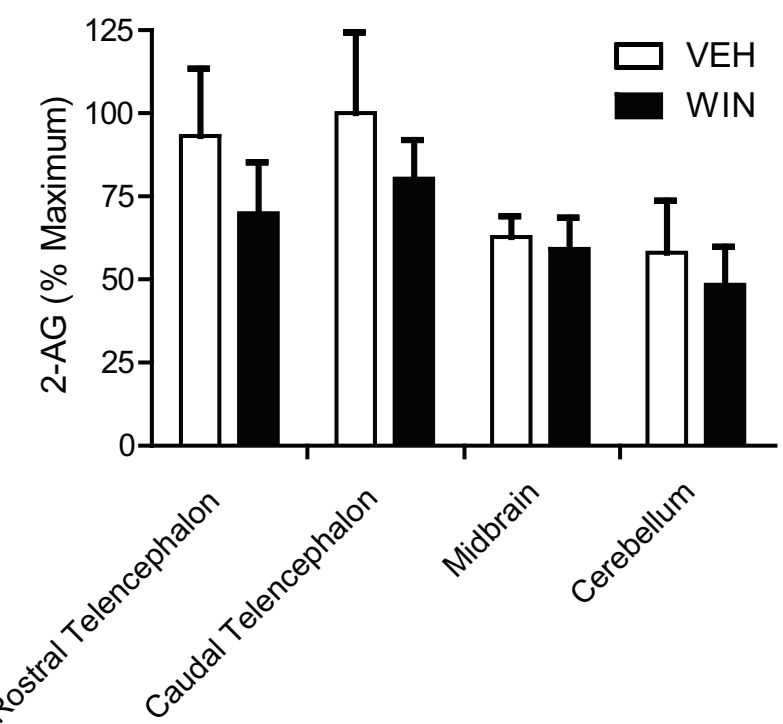

Figure 3 Effects of chronic, developmental, cannabinoid treatments on endogenous 2-AG levels in zebra finch brain. Brains were rapidly dissected into rostral (Rostral Tel.) and caudal (Caudal Tel.) telencephalon, midbrain and cerebellum. Lipids were extracted, spiked with deuterium-labeled internal standards and subjected to LC-ESI-MS-MS for quantitative analysis of 2-arachidonyl glycerol (2-AG) content. (A): ANOVA indicates a significant relationship between brain region and 2-AG content $(p<0.05)$. Post-hoc analysis reveals a significant increase in 2-AG content in rostral telencephalon (which contains the song regions IMAN and Area X). Significant decreases in endocannabinoid content within cerebellum were observed following developmental WIN ( $\left.{ }^{*} p<0.05\right)$. (B): No effect of repeated WIN treatments given during adulthood on 2-AG levels were found.

membrane-delimited and potential intracellular pools of receptor protein, making it unlikely that the rapid (90 min post-treatment) changes in staining density that we observe are due to acute cellular translocation of receptor protein (although this may very well be occurring). A more likely mechanism for rapid changes in antibody staining may involve changes in epitope access related to interaction with other cellular proteins. The peptide used to produce our anti-zebra finch $\mathrm{CB}_{1}$ antibody represents the first 16 amino acids of the intracellular tail region of the receptor [3], a domain implicated in signal transduction and interaction with intracellular regulatory proteins [11]. Fixation and cross-linking of tissue with receptors coupled to such regulatory or signal transduction proteins may occlude epitope access, reducing antibody interaction and resulting in reduced staining intensity. Thus, differences in staining densities that we have documented may be more indicative of differential effects on $\mathrm{CB}_{1}$ interaction with other proteins than on receptor abundance itself. Of course we cannot exclude the possibility that decreased immunostaining may indicate receptor degradation and/or reduced expression, as both phenomenon are known to occur following agonist activation [10].

It is interesting that acute WIN treatment of birds chronically treated with vehicle control injections responded differently following late-postnatal and adult treatments (for example, see VEH-WIN responses in Figures $1 \mathrm{~B}$ and $2 \mathrm{~B}$ ). We observed similar differential effects of control treatments on acute changes in FoxP2 expression [12]. These differences may be attributable to distinct sensitivity to handling stress during sensorimotor development, but not adulthood. Alternatively, a differential sensitivity to visual isolation in developing vs. adult animals may have contributed. Following developmental treatment, birds were housed in visual isolation to prevent potential song learning from other subjects (animals treated as adults were also isolated for the same period as part of the control treatment). This isolation lasted 25 days, encompassing late-sensorimotor development to early adulthood (75-100 days). Zebra finches are social, flocking birds, and so isolation during development may have produced stress [13]. The potential for handling- and isolation-induced effects on vocal development is a question worthy of additional study. It is notable that accumulating evidence supports a role for endocannabinoid signaling in stress responses and fear extinction [14].

Despite the confounding effects of control treatments, chronic effects of repeated WIN exposure on later acute WIN sensitivity are clear. Following developmental WIN treatment, staining densities were reduced in all of the 
brain regions except DLM (Figure 1, compare VEHVEH to WIN-VEH). Following adult treatments, significant increases were seen in only three regions: HVC, RA, and DLM. HVC and RA are vocal motor regions of caudal telencephalon (see Figure 4). Activity in HVC drives RA which outputs to the nucleus of the twelfth cranial nerve which innervates the syrinx, eliciting vocalization [15]. The thalamic region, DLM, modulates this caudal vocal-motor output indirectly through projection to the rostral song region, IMAN, that in-turn, projects to RA [16]. Distinct cannabinoid effects in these vocalmotor regions during adulthood further suggests a normative role for cannabinoid signaling in song production, a conclusion reinforced by earlier evidence that acute WIN decreases vocal output in adults [17]. In addition to modulating vocal output in adulthood, the rostral song regions IMAN and Area $\mathrm{X}$ are essential for vocal learning during development [18]. More generalized effects within all song regions but DLM following developmental cannabinoid exposure, underscores the distinct sensitivity to cannabinoid agonism during periods of zebra finch vocal learning.

On the surface it appears that developmental exposure-related increased levels of the endocannabinoid, 2-AG, and decreased $\mathrm{CB}_{1}$ immunostaining are contradictory (compare Figure $3 \mathrm{~A}$ and Figure $1 \mathrm{~A}$ and $1 \mathrm{C}$ ). However, if as proposed above, staining is more a function of receptor state than abundance, it follows that persistently decreased staining following developmental agonist exposure may be due to interaction with signal transduction proteins that antagonize binding of the antibody. Such coupling may actually increase the

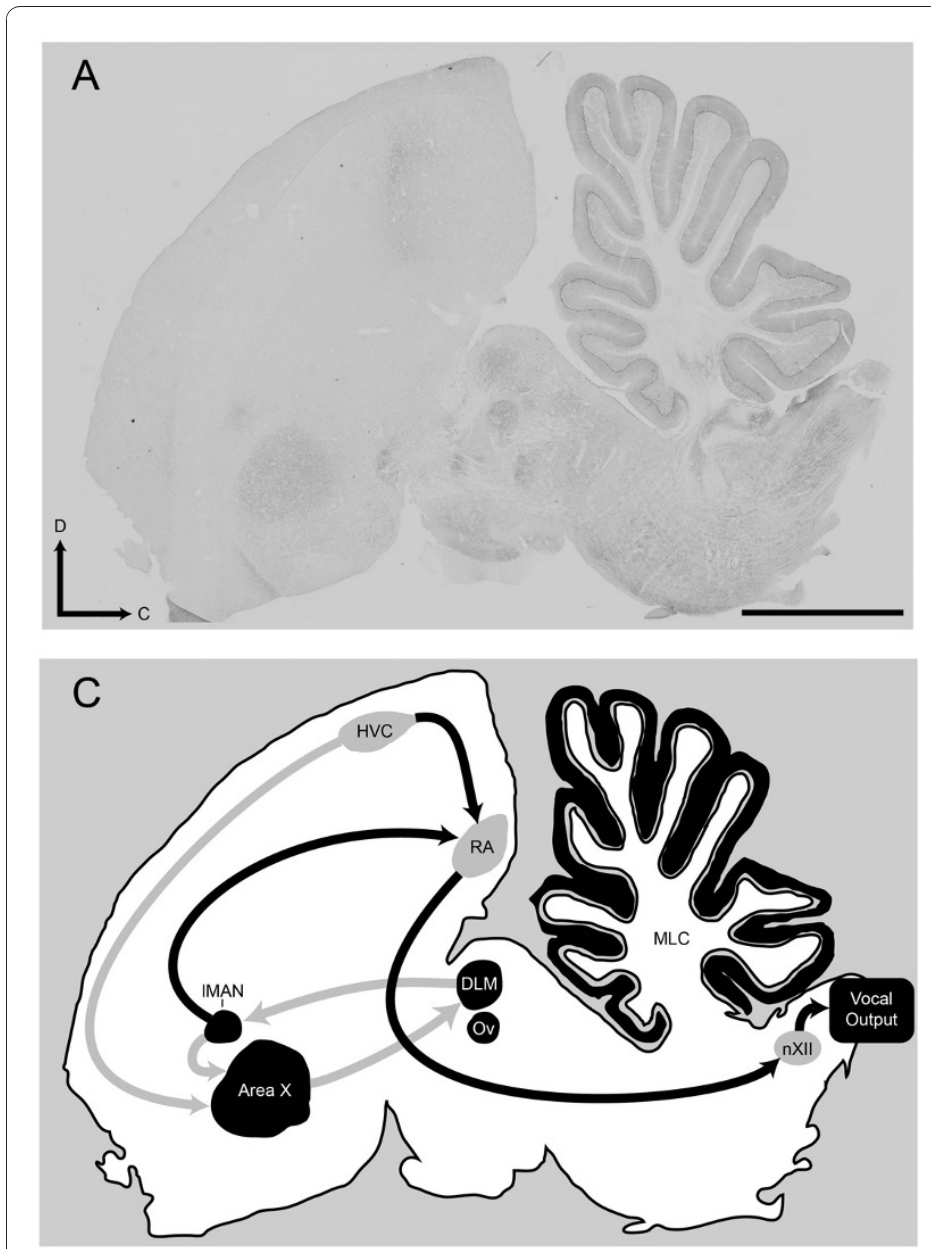

Figure 4 Representative CB1 immunostaining. (A) Medial parasagittal sections contain rostral song regions IMAN and Area $X$, thalamic regions DLM and OV, and cerebellum. (B) More lateral parasagittal sections capture HVC and RA. Dorsal and caudal are indicated by arrows, the bars $=1 \mathrm{~mm}$. (C) A tracing of the micrograph in panel A serves as a diagram summarizing relative locations of song regions studied. Regions present in panel A are represented in black, other regions are diagrammatically represented in grey (HVC, RA and nXII). Established interconnections between song regions are indicated with arrows. Grey arrows indicate rostral forebrain circuitry essential for vocal learning. Black arrows indicate caudal vocal-motor circuitry. 
population of functional $\mathrm{CB}_{1}$ receptors. If this is the case, then acute increases in staining following WIN challenge of animals developmentally exposed may indicate uncoupling from other cellular proteins and decreased responsiveness (compare WIN-VEH and WIN-WIN groups in all panels of Figure 1, note that similar increases were not seen following adult treatment, Figure 2). Assays of $\mathrm{CB}_{1}$ receptor function following developmental treatments will be required to clearly test this hypothesis [19].

Reports of rapid changes in expression of endocannabinoid signaling elements in vivo have not been widely reported, and this is a key contribution of the work reported herein. Most prior reports of altered expression of cannabinoid signaling elements in vivo involve studies of regulation of feeding behavior. In zebra finches, we have found that brief periods of food deprivation are associated with increased brain levels of 2-AG [3]. In pancreatic islet cells of Wistar rats, overnight periods of food deprivation are associated with increased expression of $\mathrm{CB}_{1}$ receptors, an effect rapidly reversed by oral glucose [20].

Unlike food restriction-induced general increases of 2AG levels in all regions of brain except cerebellum [3], developmental WIN exposure increased 2-AG selectively within rostral telencephalon and decreased levels in cerebellum (Figure 3A). These results indicate that cannabinoid-altered vocal development is associated with persistent enhancement of endocannabinoid signaling within rostral telencephalon. This is interesting as the song regions of rostral telencephalon (IMAN and Area $\mathrm{X}$, see Figure 4), are not essential for production of learned song $[21,22]$, and seem to perform a modulatory function related to song timing, that perhaps serves as an error-correcting mechanism [16]. Decreased 2-AG content within cerebellum is notable, as this region has not typically been associated with vocal development. Potential persistent effects of developmental cannabinoid exposure on motor function warrants further investigation, and recent evidence suggests that cerebellum is more important to sensory integration and cognition in zebra finches than previously thought [23]. In mice, functional changes in cerebellar sensitivity to cannabinoid drugs have been observed as a function of tolerance and following antagonist-precipitated withdrawal [24]. This raises the possibility that similar effects of chronic treatment are produced following cannabinoidaltered vocal learning, and may contribute to altered developmental course.

Production of opposing effects on 2-AG levels across CNS regions by the same drug is interesting, and may be attributable to differing neurochemistry within each area (e.g. rostral telencephalon vs. cerebellum). A growing consensus supports a presynaptic inhibitory role for endocannabinoid activation of $\mathrm{CB}_{1}$ to reduce the probability of neurotransmitter release. Because presynaptic $\mathrm{CB}_{1}$ activation inhibits release of both excitatory (e.g. glutamatergic) and inhibitory (e.g. GABAergic) transmitters, regional cannabinoid effects may depend upon relative levels of excitatory vs. inhibitory tone (reviewed by [25]). For example, across all species studied, $\mathrm{CB}_{1}$ densities are particularly high within the molecular layer of cerebellum [26]. This region is largely comprised of the axonal parallel fibers that rise from cerebellar granule cells. These parallel fibers synapse on Purkinje cell dendritic arbors, primarily releasing excitatory glutamate (reviewed by [27]). Presynaptic $\mathrm{CB}_{1}$ activation produced by repeated WIN treatments would be expected to reduce the probability of excitatory glutamate release, effectively reducing neural activity, and decreasing postsynaptic release of 2-AG. In the case of rostral telencephalon, distinct and dense $C_{1}$ receptors are expressed within the prominent Area $\mathrm{X}$ of striatum [7]. $\mathrm{CB}_{1}$ expression is particularly dense within Area $X$ at 50-75 days of age, the period during which exogenous WIN treatments were administered in our current studies [28]. In contrast to molecular layer of cerebellum, Area X is characterized by significant inhibitory GABAergic transmission $[29,30]$. Thus, cannabinoid agonism may be expected to reduce inhibitory signaling within Area X, leading to increased neuronal activity and post-synaptic 2-AG release.

\section{Conclusions}

In summary, repeated cannabinoid agonist exposure during zebra finch sensorimotor vocal development was associated with increased levels of the endocannabinoid, 2-AG, and decreased $C_{1}$ immunostaining intensities within regions of rostral telencephalon. Similar changes were not observed following repeated WIN treatment of adult animals, demonstrating distinct developmental cannabinoid sensitivity during vocal development. Developmental WIN exposure also altered acute responsiveness to WIN challenge, an effect that was not seen following chronic treatment of adults. These findings suggest that the cannabinoid-altered vocal development involves persistent changes in expression, regulation and responsiveness of endocannabinoid signaling elements. In the context of the developing zebra finch CNS, these endocannabinoid signalling elements are particularly susceptible to disregulation caused by prolonged exposure to cannabinoids.

\section{Methods}

\section{Animals}

Male zebra finches bred in our aviary and sexed at $\sim 25$ days via PCR [31] were used in these experiments. Except where indicated otherwise, six animals were 
assigned to each treatment group. Prior to the start of experiments, birds were housed with an adult male song tutor in flight aviaries and provided free access to mixed seeds (SunSeed VitaFinch), grit, water, and cuttlebone. Each flight aviary contained several perches. The lightdark cycle was controlled at L:D 14:10 h and ambient temperature was maintained at $78^{\circ} \mathrm{F}$.

Animals were cared for and experiments conducted according to protocols approved by East Carolina University's Animal Care and Use Committee.

\section{Treatments}

Drug treatments were given by intramuscular injection of $50 \mu \mathrm{l}$ into the pectoralis muscle. Drug dilutions for injection were made from $10 \mathrm{mM}$ stocks (in DMSO) to produce a final vehicle of 1:1:18 DMSO:Alkamuls (Rhodia, Cranberry, NJ):PBS $(\mathrm{pH}=7.4)$. Because zebra finches are inactive and don't sing in the dark, treatments were given immediately prior to the beginning of light cycles to avoid potential song- and activity-related changes in receptor expression. Prior work investigating immediate early gene expression experiments indicated that peak agonist-induced immunoreactivity of both ZENK and FoxP2 occurs 90 min following treatment with the potent $\mathrm{CB}_{1} / \mathrm{CB}_{2}$ receptor antagonist WIN55,212-2 (WIN) and therefore this period was adopted to investigate potential acute changes in $\mathrm{CB}_{1}$ immunostaining [32,33].

For developmental experiments, once-daily injections of vehicle (VEH- groups) or $1 \mathrm{mg} / \mathrm{kg}$ WIN (WINgroups) were given to male zebra finches from 50 to 75 days of age (during the sensorimotor period of zebra finch vocal learning). WIN treatment during this period is well-documented to alter both song stereotypy and incorporation of notes into mature song (see [34,35]). Following completion of treatments, animals were allowed to mature to at least 100 days of age in visual isolation. Upon maturation, groups of animals were either given a single acute vehicle injection (VEH-VEH and WIN-VEH groups), or given a single acute injection of $3 \mathrm{mg} / \mathrm{kg}$ WIN in order to induce potential changes in $\mathrm{CB}_{1}$ immunoreactivity (VEH-WIN and WIN-WIN). Groups of adults $(n=4)$ were treated similarly to control for effects dependent on developmental exposure. Note that treatment groups are designated by the repeated, developmental treatment indicated first, and separated by a hyphen from the indication of a second final acute treatment given in adulthood. Treatment groups and designations are summarized for clarity in Table 1.

\section{$\mathrm{CB}_{1}$ immunostaining}

Ninety minutes following acute treatments, birds were killed by Equithesin overdose and transcardially perfused
Table 1 Summary of Treatment Groups

\begin{tabular}{cccc}
\hline Abbreviation & \# Animals & $\begin{array}{c}\text { Chronic Tx } \\
\text { (QD for 25 days) }\end{array}$ & $\begin{array}{c}\text { Acute Tx } \\
\text { 1× in adulthood }\end{array}$ \\
\hline VEH-VEH & $6 \mathrm{LPN}^{*}$, 4 Adult & Vehicle & Vehicle \\
VEH-WIN & $6 \mathrm{LPN}^{*}$, 4 Adult & Vehicle & $3 \mathrm{mg} / \mathrm{kg} \mathrm{WIN}$ \\
WIN-VEH & $6 \mathrm{LPN}^{*}, 4$ Adult & $1 \mathrm{mg} / \mathrm{kg} \mathrm{WIN}$ & Vehicle \\
WIN-WIN & $6 \mathrm{LPN}^{*}, 4$ Adult & $1 \mathrm{mg} / \mathrm{kg} \mathrm{WIN}$ & $3 \mathrm{mg} / \mathrm{kg} \mathrm{WIN}$ \\
\hline
\end{tabular}

*LPN, Late-Postnatal Treatment.

with phosphate-buffered saline (PBS, $\mathrm{pH}=7.4$ ) followed by phosphate-buffered $4 \%$ paraformaldehyde, $\mathrm{pH}=7.0$. After brains were removed and immersed overnight in buffered $4 \%$ paraformaldehyde, they were blocked down the midline and left hemispheres were sectioned parasagittally (lateral to medial) on a vibrating microtome. Immunohistochemistry was performed using a standard protocol reported in [36]. For immunohistochemistry experiments, 30 micron sections of zebra finch brain were reacted with a 1:3000 dilution of polyclonal antizebra finch $\mathrm{CB}_{1}$ antibody raised in rabbit. The selectivity of this antibody for zebra finch $\mathrm{CB}_{1}$ has been demonstrated previously, and it has been used in multiple studies $[3,4,37]$. Tissue sections were rinsed in $0.1 \% \mathrm{H}_{2} \mathrm{O}_{2}$ for $30 \mathrm{~min}$, blocked with $5 \%$ goat serum for $30 \mathrm{~min}$, and reacted overnight in blocking solution containing antizebra finch $\mathrm{CB}_{1}$ antibody (1:3000). After antibody exposure, sections were rinsed in PBS $(\mathrm{pH}=7.4)$, incubated in blocking solution containing biotinylated goat antirabbit antiserum (1:500) for 1 hour, rinsed with PBS again, and then submerged in avidin-biotin-peroxidase complex solution (purchased as a kit from Vector Laboratories, cat \# PK-4005) for 1 hour. Antibody labelling was visualized with DAB solution (Vector cat \# SK-4100). Control sections that were not reacted in primary antibody were not immunoreactive. To eliminate possible variance associated with reaction conditions, tissue from equal numbers of animals from each treatment group within an experiment were processed simultaneously.

Staining was examined in various brain regions at $40 \times$ using an Olympus BX51 microscope under brightfield conditions. Multiple images were captured using a Spot Insight QE digital camera and Image-Pro Plus software (MediaCybernetics, Silver Spring, MD) under identical, calibrated exposure conditions. These images were background-corrected and converted to grey scale (see Figure 4). The borders of brain regions were traced manually. In cerebellum, only molecular layer staining was measured. Mean optical densities of brain areas enclosed within traced regions were determined without knowledge of treatment condition from five separate sections per animal using Image-Pro Plus software. Measurements were made independently by two investigators 
and pooled for analysis. Mean optical densities within each region were compared across treatment group using one-way ANOVA as described below.

\section{Determination of 2-arachidonylglycerol (2-AG) content} Groups of male zebra finches were randomly assigned to receive vehicle or $1 \mathrm{mg} / \mathrm{kg}$ WIN injections from 50 to 75 days of age $(\mathrm{n}=4$ animals per treatment). Following these developmental treatments, birds were allowed to mature to adulthood (100 days of age) in visual, but not auditory isolation. To assess dependence of effects on developmental exposure a separate experiment was done with adult animals $(n=4)$ employing a similar 25-day treatment period followed by visual isolation. Following treatment and maturation, birds were killed by anesthetic overdose and brains rapidly removed to ice. Brains were blocked down the midline, and tissue from each hemisphere was extracted and analyzed independently resulting in two data points per sample per bird. Developmental experiments were performed in triplicate resulting in a final $\mathrm{n}=24$ per treatment. Tissue from each brain half was rapidly dissected into: rostral (containing the song regions IMAN [lateral magnocellular nucleus of anterior nidopallium] and Area X of striatum); and caudal (containing the song regions HVC and RA [robust nucleus of arcopallium] and auditory regions L2 and NCM) telencephalon; cerebellum; and midbrain and immediately frozen in liquid nitrogen. Frozen tissue was stored at $-80^{\circ} \mathrm{C}$ until extracted as described previously (Matias et al., 2003). Briefly, tissues were homogenized in and extracted with chloroform:methanol:Tris$\mathrm{HCl} 50 \mathrm{mM}, \mathrm{pH}=7.5,2: 1: 1,(\mathrm{v} / \mathrm{v})$ containing internal standards (10 pmol of anandamide- $\mathrm{d} 8$, palmitoylethanolamine-d4 and $100 \mathrm{pmol}$ of 2-arachidonyl glycerol-d5, obtained from Cayman Chemicals). The lipid-containing organic phase was collected and dried. Samples were reconstituted in $100 \mu \mathrm{L}$ of 10:90 (v/v) water: methanol with $0.1 \%$ ammonium acetate and placed in autosample vials for analysis.

The electrospray ionization-mass spectrometry-mass spectrometry (LC-ESI-MS-MS) method used to detect and quantitative anandamide and 2-arachidonyl glycerol (2-AG) was a modification of a previously published method [38]. The LC-ESI-MS-MS system used was Shimadzu Shumadzu Prominence LC system coupled to an Applied Bio Systems 3200 Q trap with a turbo V source for TurbolonSpray. The MS analyses were carried out in the multiple reaction monitoring mode and the following transition ions were monitored: $(348>62)$ and $(348$ $>91)$ for anandamide; $(356>62)$ for Anandamide -d8; $(379>287)$ and $(279>269)$ for 2-AG; $(384>96)$ for 2AG-d5. The analytical column used was a Discovery ${ }^{\circledR}$ HS C18, $4.6 \mathrm{~mm} \times 15 \mathrm{~cm}, 3$ micron (Supelco, USA). The mobile phase consisted of (10:90) water: methanol with $0.1 \%$ ammonium acetate and $0.1 \%$ formic acid and a flow rate of $0.3 \mathrm{~mL} / \mathrm{min}$ was used. A negative control and seven point calibration curves at concentrations of $0,0.25,0.5,1,2,4,8$ pmoles for 2-AG and 0, 0.038, $0.075,0.15,0.3,0.6$, and 1.2 pmoles for anandamide were prepared with each analytical run. Sample concentrations were calculated by linear regression.

\section{Statistical Analyses}

Because sections containing all brain regions were reacted together, and each antibody reaction contained tissue from all four treatment groups, relationships between drug treatments and within-region $\mathrm{CB}_{1}$ immunostaining optical densities were determined through two-way ANOVA by treatment and brain region. Following ANOVA determination that mean relative optical density values differed across treatment, Student-Newman-Keuls post-tests were done to determine brain regions with that differed by treatment. In the case of 2-AG measurements, brain areas were processed independently, and therefore mean content of each area by vehicle or WIN treatment groups were compared using t-tests.

\section{Acknowledgements}

The authors are grateful for the excellent technical assistance of Bin Luo and Hoda Khafaga. This work was supported by grants (R01DA020109, R01DA015683, P01DA009789 and P50DA005274) from the National Institute on Drug Abuse.

\section{Author details}

Department of Pharmacology and Toxicology, Brody School of Medicine, East Carolina University, Greenville, NC 27834, USA. ²Department of Pharmacology and Toxicology, Virginia Commonwealth University, Richmond, Virginia 23298, USA.

\section{Authors' contributions}

KS was responsible for designing and conducting the immunohistochemical experiments presented. AHL and JLP contributed to the design of 2-AG assay experiments, which were conducted by JLP. All authors have read and approve the final manuscript.

Received: 3 September 2010 Accepted: 6 January 2011 Published: 6 January 2011

\section{References}

1. Bottjer SW, Johnson F: Circuits, hormones, and learning: vocal behavior in songbirds. J Neurobiol 1997, 33(5):602-18

2. Soderstrom K, Johnson F: Cannabinoid exposure alters learning of zebra finch vocal patterns. Brain Res Dev Brain Res 2003, 142(2):215-7.

3. Soderstrom $\mathrm{K}$, et al: Endocannabinoids link feeding state and auditory perception-related gene expression. J Neurosci 2004, 24(44):10013-21.

4. Soderstrom K, Tian Q: Developmental pattern of CB1 cannabinoid receptor immunoreactivity in brain regions important to zebra finch (Taeniopygia guttata) song learning and control. J Comp Neurol 2006, 496(5):739-758.

5. De Petrocellis L, Di Marzo V: An introduction to the endocannabinoid system: from the early to the latest concepts. Best Pract Res Clin Endocrinol Metab 2009, 23(1):1-15.

6. Jalink K, Moolenaar WH: G protein-coupled receptors: the inside story. Bioessays 2010, 32(1):13-6.

7. Soderstrom K, Tian Q: Distinct periods of cannabinoid sensitivity during zebra finch vocal development. Brain Res Dev Brain Res 2004 153(2):225-32 
8. Blair RE, et al: Prolonged exposure to WIN55,212-2 causes downregulation of the $\mathrm{CB} 1$ receptor and the development of tolerance to its anticonvulsant effects in the hippocampal neuronal culture model of acquired epilepsy. Neuropharmacology 2009, 57(3):208-218.

9. Spear L: Modeling adolescent development and alcohol use in animals. Alcohol Res Health 2000, 24(2):115-23.

10. Hsieh C, et al: Internalization and recycling of the CB1 cannabinoid receptor. J Neurochem 1999, 73(2):493-501.

11. Smith $T H$, Sim-Selley $L$, Selley DE: Cannabinoid CB1 receptor-interacting proteins: novel targets for central nervous system drug discovery? $\mathrm{Br} J$ Pharmacol 2010, 160(3):454-66.

12. Soderstrom K, Luo B: Late-postnatal cannabinoid exposure persistently increases FoxP2 expression within zebra finch striatum. Dev Neurobiol 2010, 70(3):195-203.

13. Zann R: The zebra finch: a synthesis of field an laboratory studies New York: Oxford University Press; 1996.

14. Lafenetre $P$, Chaouloff F, Marsicano G: The endocannabinoid system in the processing of anxiety and fear and how CB1 receptors may modulate fear extinction. Pharmacol Res 2007, 56(5):367-81.

15. McCasland JS: Neuronal control of bird song production. J Neurosci 1987, 7(1):23-39.

16. Leblois A, et al: Millisecond timescale disinhibition mediates fast information transmission through an avian basal ganglia loop. J Neurosci 2009, 29(49):15420-33

17. Soderstrom K, Johnson F: Zebra finch CB1 cannabinoid receptor: pharmacology and in vivo and in vitro effects of activation. J Pharmacol Exp Ther 2001, 297(1):189-97.

18. Scharff C, Nottebohm F: A comparative study of the behavioral deficits following lesions of various parts of the zebra finch song system: implications for vocal learning. J Neurosci 1991, 11(9):2896-913.

19. Nguyen PT, Selley DE, Sim-Selley LJ: Statistical Parametric Mapping reveals ligand and region-specific activation of G-proteins by CB1 receptors and non-CB1 sites in the 3D reconstructed mouse brain. Neuroimage 2010, 52(4):1243-51.

20. Vilches-Flores $A$, et al: $C B 1$ cannabinoid receptor expression is regulated by glucose and feeding in rat pancreatic islets. Regul Pept 2010, 163(13):81-7.

21. Bottjer SW, Miesner EA, Arnold AP: Forebrain lesions disrupt development but not maintenance of song in passerine birds. Science 1984, 224(4651):901-903.

22. Sohrabji F, Nordeen EJ, Nordeen KW: Selective impairment of song learning following lesions of a forebrain nucleus in the juvenile zebra finch. Behav Neural Biol 1990, 53(1):51-63.

23. Spence RD, et al: Recovery of motor and cognitive function after cerebellar lesions in a songbird: role of estrogens. Eur J Neurosci 2009, 29(6):1225-34.

24. Hutcheson DM, et al: Behavioural and biochemical evidence for signs of abstinence in mice chronically treated with delta-9tetrahydrocannabinol. Br J Pharmacol 1998, 125(7):1567-77

25. Schlicker E, Kathmann M: Modulation of transmitter release via presynaptic cannabinoid receptors. Trends Pharmacol Sci 2001, 22(11):565-572.

26. Elphick MR, Egertova M: The neurobiology and evolution of cannabinoid signalling. Philos Trans R Soc Lond B Biol Sci 2001, 356(1407):381-408.

27. Arenz A, Bracey EF, Margrie TW: Sensory representations in cerebellar granule cells. Curr Opin Neurobiol 2009, 19(4):445-451.

28. Soderstrom K, Tian Q: Developmental pattern of CB1 cannabinoid receptor immunoreactivity in brain regions important to zebra finch (Taeniopygia guttata) song learning and control. J Comp Neurol 2006, 496(5):739-58.

29. Grisham W, Arnold AP: Distribution of GABA-like immunoreactivity in the song system of the zebra finch. Brain Res 1994, 651(1-2):115-122.

30. Luo M, Perkel DJ: Long-range GABAergic projection in a circuit essential for vocal learning. J Comp Neurol 1999, 403(1):68-84.

31. Soderstrom $\mathrm{K}$, Oin $\mathrm{W}$, Leggett $\mathrm{MH}$ : A minimally invasive procedure for sexing young zebra finches. J Neurosci Methods 2007, 164(1):116-9.

32. Soderstrom K, Tian Q: $\mathrm{CB}(1)$ cannabinoid receptor activation dose dependently modulates neuronal activity within caudal but not rostral song control regions of adult zebra finch telencephalon. Psychopharmacology (Berl) 2008, 199(2):265-73.
33. Soderstrom K, Luo B: Late-postnatal cannabinoid exposure persistently increases FoxP2 expression within zebra finch striatum. Developmental Neurobiology 2010, 70(3):195-203

34. Soderstrom $\mathrm{K}$, Johnson F: Cannabinoid exposure alters learning of zebra finch vocal patterns. Brain Res Dev Brain Res 2003, 142(2):215-7.

35. Soderstrom K, Tian Q: Distinct periods of cannabinoid sensitivity during zebra finch vocal development. Developmental Brain Research 2004, 153(2):225-232.

36. Whitney $\mathrm{O}$, Soderstrom $\mathrm{K}$, Johnson F: Post-transcriptional regulation of zenk expression associated with zebra finch vocal development. Brain Res Mol Brain Res 2000, 80(2):279-90.

37. Nagel Kl, Doupe AJ: Organizing Principles of Spectro-Temporal Encoding in the Avian Primary Auditory Area Field L. Neuron 2008, 58(6):938-955.

38. Richardson D, et al: Quantitative profiling of endocannabinoids and related compounds in rat brain using liquid chromatography-tandem electrospray ionization mass spectrometry. Anal Biochem 2007. 360(2):216-26

doi:10.1186/1471-2202-12-3

Cite this article as: Soderstrom et al:: Cannabinoid exposure during zebra finch sensorimotor vocal learning persistently alters expression of endocannabinoid signaling elements and acute agonist responsiveness. BMC Neuroscience 2011 12:3.

\section{Submit your next manuscript to BioMed Central and take full advantage of:}

- Convenient online submission

- Thorough peer review

- No space constraints or color figure charges

- Immediate publication on acceptance

- Inclusion in PubMed, CAS, Scopus and Google Scholar

- Research which is freely available for redistribution

Submit your manuscript at www.biomedcentral.com/submit
C Biomed Central 